\title{
The dispersions of estimates of sensitivity obtained from four psychophysical procedures: Implications for experimental design
}

\author{
MICHAEL J. HAUTUS and ALAN J. LEE \\ University of Auckland, Auckland, New Zealand
}

\begin{abstract}
The dispersions of estimates of sensitivity obtained from the yes-no, two-alternative forced-choice (2AFC), matching-to-sample, and same-different tasks were examined to determine which task would be more appropriate to use in a given experimental context. Consideration was given to the effects of corrections for extreme sampled proportions. These corrections result in biased estimators, and hence the mean-square deviation of the sampled values about the population mean $\left[\operatorname{MSD}\left(\hat{d}^{\prime}\right)\right]$, rather than that about the mean of the estimates [VAR $\left.\left(\hat{d}^{\prime}\right)\right]$, indicates more completely the extent of the error in the estimator. For barely discriminable events $\left(d^{\prime} \simeq 0.5\right)$, the yes-no and 2AFC tasks had the lowest values of $\operatorname{MSD}\left(\hat{d}^{\prime}\right)$. However, for very discriminable events $\left(d^{\prime}>3\right)$, the samedifferent and matching-to-sample tasks had lower values of $\operatorname{MSD}\left(\hat{d}^{\prime}\right)$.
\end{abstract}

Given the variety of psychophysical tasks that are available, what criterion can be used when one is deciding which specific task to use in a given situation? There are several ways of answering this question. Macmillan and Creelman (1991, pp. 175-178) have outlined two possible sets of criteria: those based on detection-theory factors and those based on processing differences.

A detection-theory criterion would be to choose tasks, on the basis of known detection-theoretic models, that would be unlikely to produce floor or ceiling effects. For example, in a context where true $d^{\prime}$ was 0.5 , an estimate of $d^{\prime}$ obtained using the same-different task would very likely encounter a floor effect, whereas a two-alternative forcedchoice (2AFC) task would not. On the other hand, if true $d^{\prime}$ was 3.0, estimates of $d^{\prime}$ obtained using the 2AFC task would exhibit a ceiling effect, but the same-different task would not have this problem.

Criteria based on processing differences stem from empirical studies. For example, it has been found that singleinterval tasks tend to yield smaller estimates of $d^{\prime}$ than do multiple-interval tasks (see, e.g., Jesteadt \& Bilger, 1974). However, for multiple-interval tasks, estimates of $d^{\prime}$ are also dependent on the interstimulus interval (see, e.g., Berliner \& Durlach, 1973).

Another useful criterion for deciding which psychophysical task to employ in a given context is the efficiency of the task in that context. Efficiency could be defined as the

Special acknowledgment is due Howard Kaplan and Neil Macmillan for their valuable comments during the review process. Thanks also to an anonymous reviewer. This research was partially funded by a grant from the New Zealand Lottery Science Board. A.J.L. is in the Department of Statistics, University of Auckland. Correspondence should be addressed to M. Hautus, Department of Psychology, University of Auckland, Private Bag 92019, Auckland, New Zealand (e-mail: m.hautus(a) auckland.ac.nz). number of trials required to obtain an estimate of $d^{\prime}$ with a specified variance. Under this definition, the relative efficiency of two tasks is equal to the ratio of the number of trials required on each task to obtain a common variance. Relative efficiency can also be defined as the ratio of the variances of $d^{\prime}$ estimates obtained from two tasks when the same number of trials has been used in each.

In some contexts, the efficiency of a task in terms of the number of stimulus presentations (instead of the number of trials) would be more useful. This would be true in contexts in which large numbers of stimulus presentations are impractical or undesirable. Flavor discrimination is one such area.

\section{Nomenclature}

In this paper, we specify the efficiency of a task in terms of the dispersion of estimates of $d^{\prime}$ that would be obtained with a fixed number of trials. We have adopted the convention of denoting estimates of population parameters by placing a caret over the symbol for the parameter. For example $\hat{H}$ is an estimator of the population value of $H$. The number of trials is given as $N$. If the type of trial is relevant, a subscript is appended. For example, $N_{N}$ and $N_{S}$ indicate the number of "noise" and "signal" trials, respectively. All figures and tables are based on $N_{N}=N_{S}=N / 2$ unless otherwise indicated.

\section{Yes-No}

For this task,

$$
d^{\prime}=z(H)-z(F),
$$

where $z(\bullet)$ is the inverse-normal transform. Equation 1 assumes that the two normal distributions have unit variance. To obtain an estimate of $d^{\prime}$, experimentally determined estimates of $H$ and $F$ can be substituted in Equation 1. If $\hat{F}$ or $\hat{H}$ are extreme proportions (i.e., equal to either zero or 
one), then $\hat{d}^{\prime}$ takes on an infinite value. This difficulty arises for all models that incorporate the inverse-normal transform. Various corrections have been reported for extreme proportions. While the $1 /(2 N)$ correction is more commonly cited (e.g., Macmillan \& Kaplan, 1985; Miller, 1996), a correction often used in log-linear analysis has been shown to provide, in most situations, a less biased estimate of $d^{\prime}$ (Hautus, 1995). This "log-linear correction" involves adding 0.5 to all cells in the $2 \times 2$ contingency table and then adjusting marginal totals appropriately (see, e.g., Goodman, 1970). Thus $\hat{H}=(h+1 / 2) /\left(N_{S}+1\right)$ and $\hat{F}=$ $(f+1 / 2) /\left(N_{N}+1\right)$, where $h$ and $f$ refer to the frequency of hits and false alarms, respectively. This correction is usually applied to all data, irrespective of the presence of an extreme proportion (Snodgrass \& Corwin, 1988).

In general, the mean of the sampling distribution of $\hat{d}^{\prime}$ will converge fairly rapidly toward the population value, $d^{\prime}$, as $N$ increases. This occurs because the relative size of the correction, as a proportion of $N$, decreases as $N$ increases. This is true of either correction. The major difference between the two corrections is the extent of the sampling distribution to which they are applied. The $1 /(2 N)$ correction affects only the most extreme values of the sampling distribution, whereas the log-linear correction affects every value in the distribution.

Previous studies have employed various methods to investigate the biasing effects of corrections for extreme proportions on $\hat{d}^{\prime}$. Hautus (1995) conducted Monte Carlo simulations, whereas Miller (1996) presented a more direct method of investigating these effects. The latter approach will be used here.

If $p$ is the population proportion and a log-linear correction is applied to the sampling distribution of $\hat{p}$, then the expected value of $z(\hat{p})$ is given by

$$
\mathrm{E}(z(\hat{p}))=\sum_{x=0}^{N_{p}} z\left(\frac{x+\frac{1}{2}}{N_{p}+1}\right) b\left(x, N_{p}, p\right),
$$

where $b\left(x, N_{p}, p\right)$ is the binomial density and $N_{p}$ is the number of trials employed to estimate $p$. It follows that

$$
\begin{aligned}
\mathrm{E}\left(\hat{d}^{\prime}\right)= & \mathrm{E}(z(\hat{H}))-\mathrm{E}(z(\hat{F})) \\
= & \sum_{x=0}^{N_{s}} z\left(\frac{x+\frac{1}{2}}{N_{s}+1}\right) b\left(x, N_{s}, H\right) \\
& -\sum_{x=0}^{N_{n}} z\left(\frac{x+\frac{1}{2}}{N_{n}+1}\right) b\left(x, N_{n}, F\right) .
\end{aligned}
$$

and hence that $d^{\prime}-\mathrm{E}\left(\hat{d}^{\prime}\right)$ is equal to the bias introduced by the correction employed. ${ }^{1}$ This process can be extended to calculate the exact variance of $\hat{d}^{\prime}$ because

$$
\begin{aligned}
\operatorname{VAR}(z(\hat{p})) & =\mathrm{E}\left(z(\hat{p})^{2}\right)-\mathrm{E}(z(\hat{p}))^{2} \\
& =\sum_{x=0}^{N_{p}}\left[z\left(\frac{x+\frac{1}{2}}{N_{p}+1}\right)-\mathrm{E}(z(\hat{p}))\right]^{2} b\left(x, N_{p}, p\right),
\end{aligned}
$$

and the exact variance of $\hat{d}^{\prime}$ for the yes-no task given the application of the log-linear correction is given by

$$
\begin{aligned}
\operatorname{VAR}\left(\hat{d}^{\prime}\right)= & \sum_{x=0}^{N_{s}}\left[z\left(\begin{array}{c}
x+\frac{1}{2} \\
N_{s}+1
\end{array}\right)-\mathrm{E}(z(\hat{H}))\right]^{2} b\left(x, N_{s}, H\right) \\
& +\sum_{x=0}^{N_{n}}\left[z\left(\frac{x+\frac{1}{2}}{N_{n}+1}\right)-\mathrm{E}(z(\hat{F}))\right]^{2} b\left(x, N_{n}, F\right) .
\end{aligned}
$$

Equation 5 gives the variance of $\hat{d}^{\prime}$ without giving consideration to the bias introduced by the correction; that is, it gives the mean-square deviation (MSD) of the distribution of $\hat{d}^{\prime}$ about $\mathrm{E}\left(\hat{d}^{\prime}\right)$, not about $d^{\prime}$. The MSD about $d^{\prime}, \operatorname{MSD}\left(\hat{d}^{\prime}\right)$ would quantify both the bias and variance of $\hat{d}^{\prime}$ as an estimator of $d^{\prime}$ :

$$
\begin{aligned}
\operatorname{MSD}\left(\hat{d}^{\prime}\right)= & \sum_{x=0}^{N_{s}}\left[z\left(\frac{x+\frac{1}{2}}{N_{s}+1}\right)-z(H)\right]^{2} b\left(x, N_{s}, H\right) \\
& +\sum_{x=0}^{N_{n}}\left[z\left(\frac{x+\frac{1}{2}}{N_{n}+1}\right)-z(F)\right]^{2} b\left(x, N_{n}, F\right),
\end{aligned}
$$

where $\mathrm{z}(F)$ and $\mathrm{z}(H)$ are the inverse-normal transforms of the population parameters $F$ and $H$. This means that Equation 6 is undefined for extreme population proportionsa reasonable finding. Similar equations, presented in the Appendix, have been derived for the $1 /(2 N)$ correction for extreme proportions.

Figures 1 and 2 illustrate the effect of $N$ on $\operatorname{VAR}\left(\hat{d}^{\prime}\right)$ and $\operatorname{MSD}\left(\hat{d}^{\prime}\right)$ (thin and heavy lines, respectively, in the top panels), the relative difference between $\operatorname{VAR}\left(\hat{d}^{\prime}\right)$ and $\operatorname{MSDZ}\left(\hat{d}^{\prime}\right)$ (middle panels), and $\mathrm{E}\left(\hat{d}^{\prime}\right)$ (bottom panels). All functions are for the yes-no task, with either the log-linear correction (left three panels) or the $1 /(2 N)$ correction (right three panels). The functions in Figure 1 are those of an unbiased observer $(c=0)$, while those in Figure 2 are those of a fairly biased observer $(c=1)$. The index of bias used here, $c$, is defined as $c=0.5 \times[z(H)+z(F)]$ (see Macmillan \& Creelman, 1991, p. 33).

For the top panels, the ordinate and abscissa are scaled logarithmically. When the MSDs are proportional to $1 / N$, the functions should be linear. In every case, the functions approximate straight lines only when $\operatorname{MSD}\left(\hat{d}^{\prime}\right) \simeq \operatorname{VAR}\left(\hat{d}^{\prime}\right)$ (as seen in the middle panels). The bottom panels indicate that this occurs only when $d^{\prime} \simeq \mathrm{E}\left(\hat{d}^{\prime}\right)$, and hence bias is almost zero.

Figures 1 and 2 provide a fair basis from which to choose the most desirable correction. Three features are evident: (1) The log-linear correction exhibits better bias-reduction properties. This is shown in the top panels by the smaller number of trials required to attain linearity when the loglinear correction was employed, and in the bottom panels by the more rapid convergence of $\mathrm{E}\left(\hat{d}^{\prime}\right)$ to $d^{\prime}$ as $N$ increases for this correction. (2) The top right panel of Figure 1 indicates that $\operatorname{MSD}\left(d^{\prime}\right)$ (the thick lines) can actually increase as a function of $N$ when the $1 /(2 N)$ correction is used. This is par- 

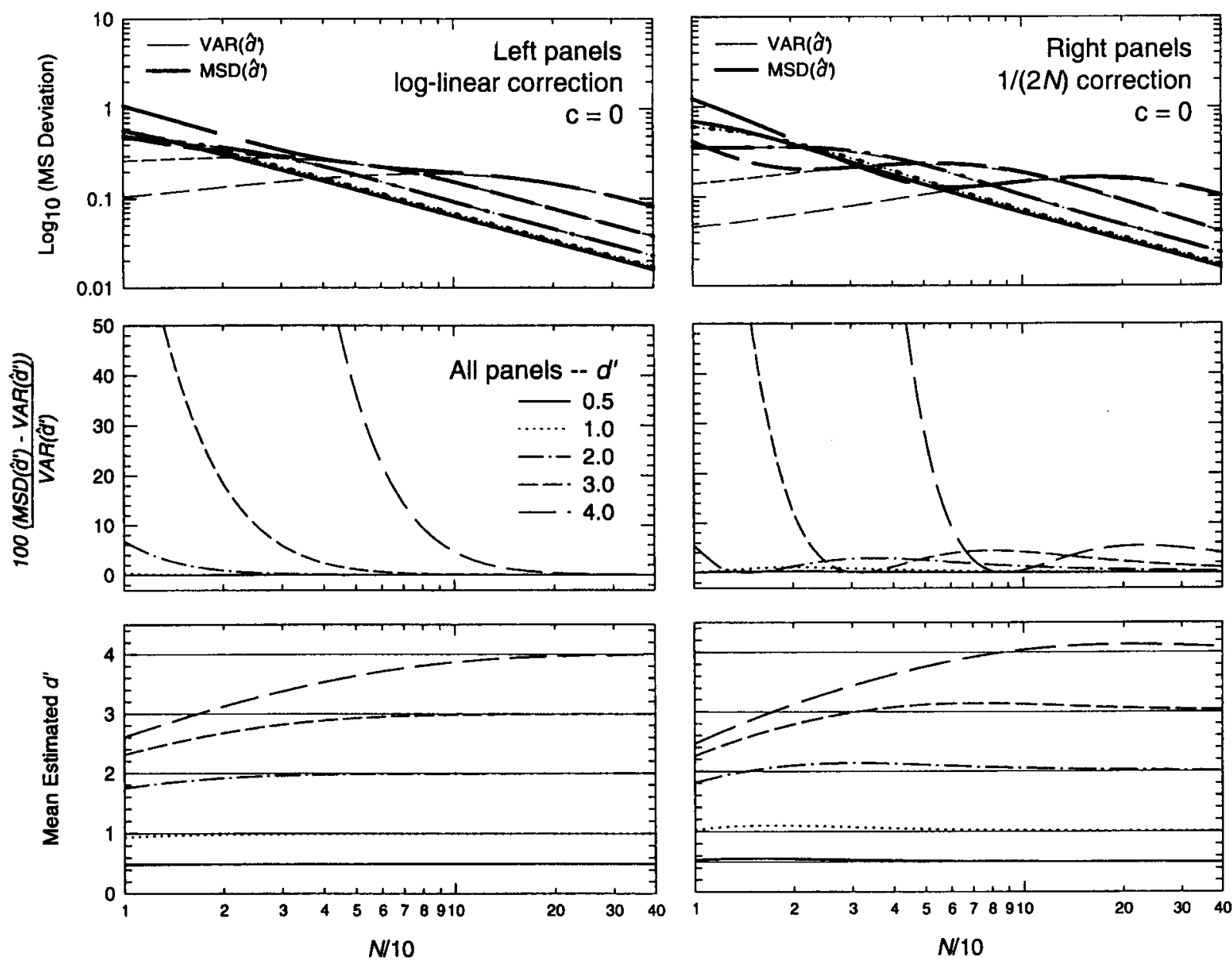

Figure 1. Values of interest presented as a function of $N$ for five different values of $d^{\prime}: 0.5,1.0,2.0,3.0$, and 4.0. The panels illustrate (top) $\operatorname{VAR}\left(\hat{d}^{\prime}\right)$ (thin lines) and MSD $\left(\hat{d}^{\prime}\right)$ (thick lines); (middle) the difference between $\operatorname{VAR}\left(\hat{d}^{\prime}\right)$ and $\operatorname{MSD}\left(\hat{d}^{\prime}\right)$, as a percentage of VAR $\left(\hat{d}^{\prime}\right)$; and (bottom) $\mathrm{E}\left(\hat{d}^{\prime}\right)$. Functions for the log-linear (left three panels) and $1 /(2 N)$ corrections (right three panels) are shown. The observer is unbiased $(c=0)$.

ticularly apparent for the case of $d^{\prime}=3.0$ and is a potentially counterproductive characteristic of the $1 /(2 N)$ correction. (3) As noted by Hautus (1995), E( $\left.\hat{d}^{\prime}\right)$ as a function of $N$ converges monotonically (from below) on $d^{\prime}$ for the log-linear correction, but converges nonmonotonically for the $1 /(2 N)$ correction. For these three reasons, only the log-linear correction will be considered for the remainder of this paper.

Figures 1 and 2 also indicate clearly that $\operatorname{VAR}\left(\hat{d}^{\prime}\right)$ is less desirable than $\operatorname{MSD}\left(\hat{d}^{\prime}\right)$ as an indicator of the efficiency of a task. For example, when $d^{\prime}=4$ in Figure 1, $\operatorname{VAR}\left(\hat{d}^{\prime}\right)$ actually increases as a function of $N$ up to about 100 trials, even when the log-linear correction is employed. This occurs because the increase in the range of the sampling distribution as $N$ increases has a larger effect on $\operatorname{VAR}\left(\hat{d}^{\prime}\right)$ than the corresponding decrease in occurrence of extreme proportions.

\section{AFC}

The evidence space for the 2AFC task is similar to that for the yes-no task except that the two normal distributions have a variance of two (Green \& Swets, 1966). Hence, for the $2 \mathrm{AFC}$ task the relationship between $d^{\prime}, F$, and $H$ is

$$
d^{\prime}=\frac{1}{\sqrt{2}}(z(H)-z(F)) \text {. }
$$

It is important to remember that, given the same events, the values of $F$ and $H$ for the 2AFC task (or any other task) will be different from the values of these proportions for the yes-no task. $\operatorname{VAR}\left(\hat{d}^{\prime}\right)$ and $\operatorname{MSD}\left(\hat{d}^{\prime}\right)$ can be obtained simply by dividing Equations 5 and 6 (or the equivalent equations for the $1 /(2 N)$ correction, which are given in the Appendix) by two.

\section{Same-Different}

The representation of the same-different task is more complicated than those of either of the two tasks already examined. The evidence space for this task consists of four bivariate-normal distributions arranged in a square with the nondiagonal distance between the means equal to $d^{\prime}$. Each dimension of the evidence space represents the evi- 

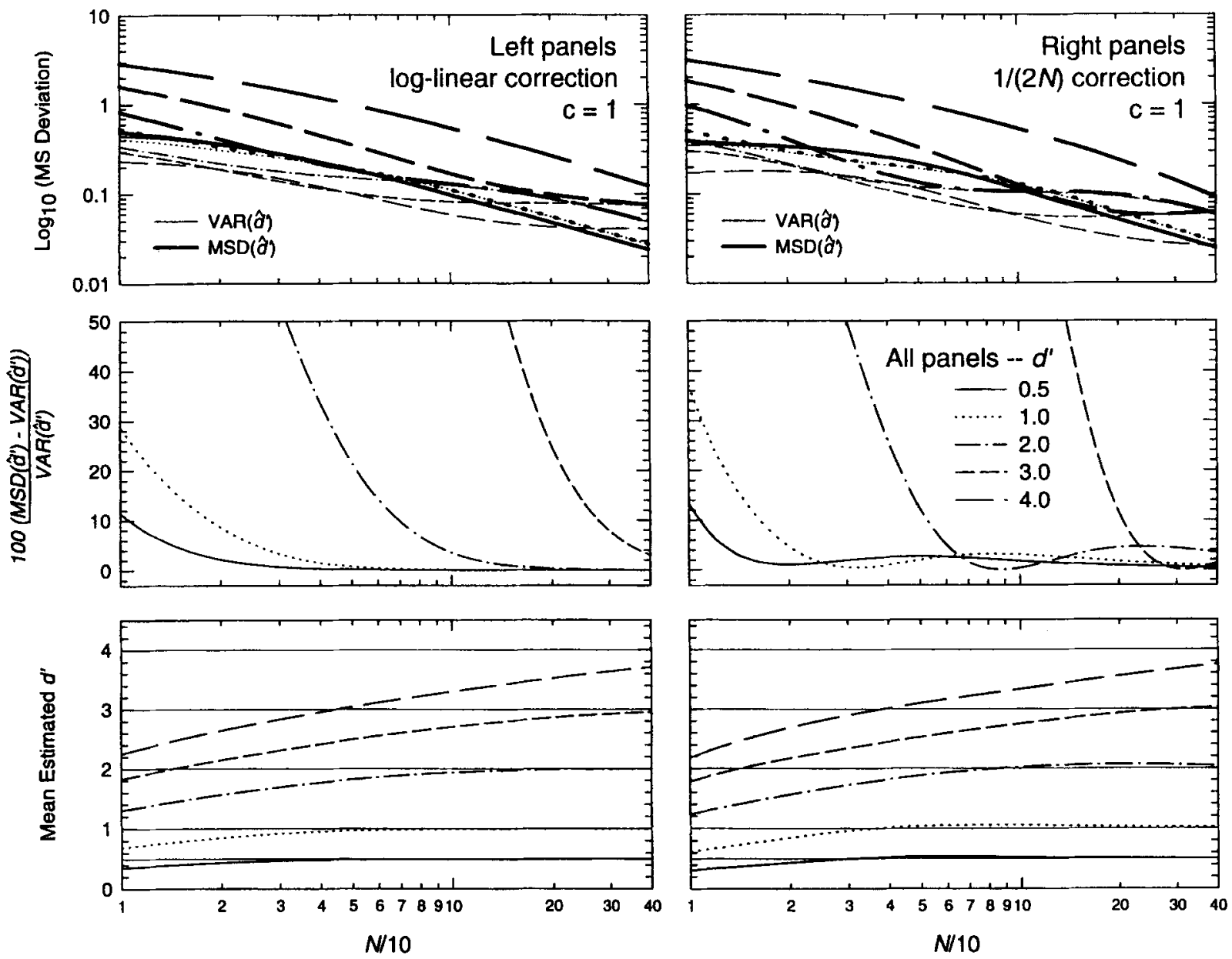

Figure 2. Values of interest presented as a function of $N$ for five different values of $d^{\prime}: 0.5,1.0,2.0,3.0$, and 4.0. The panels illustrate (top) VAR $\left(\hat{d}^{\prime}\right)$ (thin lines) and MSD $\left(\hat{d}^{\prime}\right)$ (thick lines); (middle) the difference between VAR( $\left(\hat{d}^{\prime}\right)$ and $\operatorname{MSD}\left(\hat{d}^{\prime}\right)$, as a percentage of VAR $\left(\hat{d}^{\prime}\right)$; and (bottom) $\mathrm{E}\left(\hat{d}^{\prime}\right)$. Functions for the log-linear (left three panels) and $1 /(2 N)$ corrections (right three panels) are shown. The observer is moderately biased $(c=1)$.

dence arising from one of the two independent stimulus presentations on each trial (see Noreen, 1981).

When a difference decision strategy ${ }^{2}$ is applied to the evidence space, the resulting decision space can be represented unidimensionally, as illustrated in Figure 3 (Sorkin, 1962). The decision variable, $X_{2}-X_{1}$, is the difference between the sensory evidence arising from each observation within a trial. This space consists of three normal distributions, each with a variance of 2 . The means of the different distributions are at $\pm d^{\prime}$. The decision criterion is symmetrically placed about the mean of the central distribution, which is at zero. This distribution is the evidence arising from events that are the same, and the other two distributions give the evidence arising from events that are different. Observations that give rise to evidence that falls within the criterial difference are judged same and those that give rise to evidence that falls outside of this criterial difference are judged different.

The hit rate is equal to the proportion of the area under the central distribution that falls within the criterial difference, $[-k,+k]$, and the false alarm rate is the propor- tion of the area under the other two distributions that falls within the criterial difference. If $k$ is the criterion, then

$$
H=2 \Phi\left(\frac{k}{\sqrt{2}}\right)-1
$$

and

$$
F=\Phi\left(\frac{k+d^{\prime}}{\sqrt{2}}\right)+\Phi\left(\frac{k-d^{\prime}}{\sqrt{2}}\right)-1
$$

An implicit equation ${ }^{3}$ relating $d^{\prime}$ to $F$ and $H$ can be formed and $d^{\prime}$ can be calculated for any values of $F$ and $H$ using iterative techniques. Tables have been prepared for this task (Kaplan, Macmillan, \& Creelman, 1978). The implicit equation is

$$
\Phi\left(z\left(\frac{H+1}{2}\right)+\frac{d^{\prime}}{\sqrt{2}}\right)+\Phi\left(z\left(\frac{H+1}{2}\right)-\frac{d^{\prime}}{\sqrt{2}}\right)-F-1=0 .
$$

A bivariate-binomial sampling distribution for $\hat{F}$ and $\hat{H}$ can be defined for any specified value of $N, d^{\prime}$, and $k$, and the 


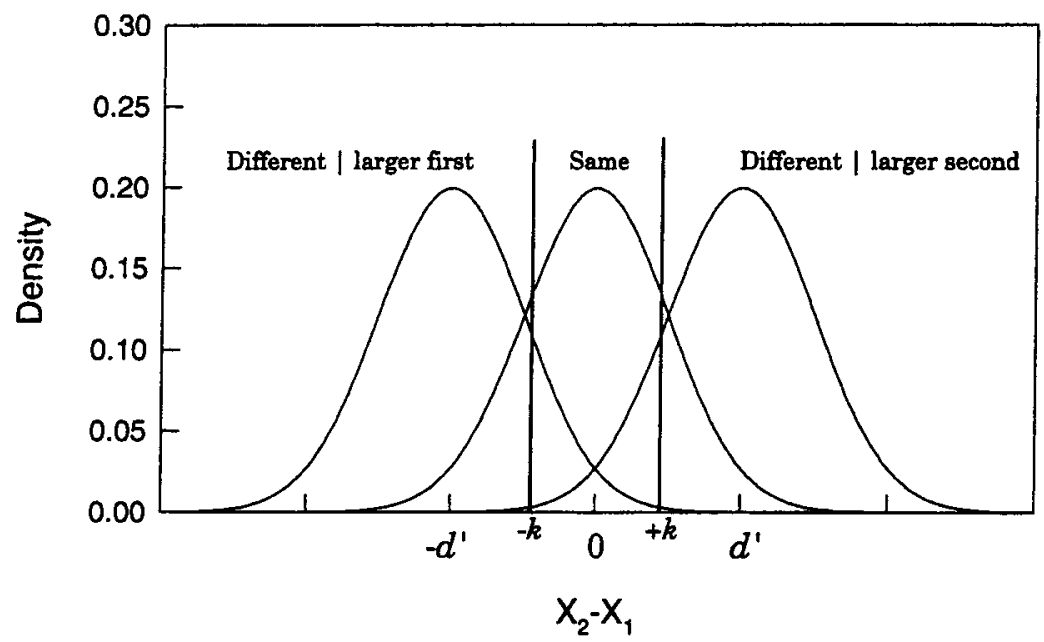

Figure 3. The decision space for the same-different task when the decision strategy is based on the difference between the sensory evidence available from each observation interval. The difference criterion is set at $[-k,+k]$. If the sensory difference falls within this region, the observer will respond "same"; otherwise, the observer will respond "different."

$\log$-linear correction can be applied to the sampling distribution. Each discrete location on the corrected bivariate distribution is associated with a particular value of $\hat{F}$ and $\hat{H}$, and consequently a particular value of $\hat{d}^{\prime}$. Hence $\operatorname{VAR}\left(\hat{d}^{\prime}\right)$ and $\operatorname{MSD}\left(\hat{d}^{\prime}\right)$ can be calculated exactly by tabulating the value of $\left(\hat{d}^{\prime}\right)$, and its probability of occurrence, at each point on the distribution and then using these tabulated values to calculate the desired statistics.

\section{Matching to Sample}

The matching-to-sample task is a variation of the $\mathrm{ABX}$ design. In this task, the observer is presented with three stimuli, the first of which is the "sample" and is presented in Interval X. The observer must judge whether the event associated with Interval $A$ or that associated with Interval $B$ is a match to the event associated with the sample. Events are randomly associated with each interval with the restriction that the events associated with Intervals A and $B$ must be different in any trial. The assumption is made that each event has an equal probability of being associated with Intervals A and B.

The evidence space for the matching-to-sample task (and other $\mathrm{ABX}$ variations) is geometrically similar to the bivariate-normal representation of the same-different task. The model presented here for this task assumes a difference decision strategy (Pierce \& Gilbert, 1958). This strategy can be compared with the optimal or likelihoodratio strategy, which, if it can be implemented by the observer, leads to better performance (see Macmillan, Kaplan, \& Creelman, 1977).

One of the dimensions, $X_{1}$, represents the difference between the evidence arising from the events occurring in Interval $\mathrm{A}$ and Interval $\mathrm{X}$; the other dimension, $X_{2}$, represents the difference between the evidence arising from the events occurring in Interval B and Interval X. Hence the two distributions have a positive covariance of 1 (Figure 4).

On each trial the observer makes a judgment, subject to his/her bias toward a particular response, about which of the two differences was smaller. The assumption is made that the judgment is based on a likelihood-ratio criterion. An unbiased observer adopts a likelihood-ratio criterion of $\beta=1$. This decision criterion, represented in Figure 4 by intersecting lines of slope \pm 1 , results in the observer choosing the interval with the smallest sensory difference from Interval X. Biased observers adopt a likelihood-ratio criterion either greater than or less than unity. Likelihoodratio criterions of $\beta=2$ and $\beta=1 / 2$ are also illustrated in Figure 4 by the long and short dashed lines, respectively.

If a false alarm is defined as the occurrence of Response $\mathrm{A}$ when the correct response was $\mathrm{B}$, then the false alarm rate is equal to the proportion of the volume under the distributions with centroids at $X_{1}=-d^{\prime}$ and $X_{1}=d^{\prime}$ that falls within the regions in Figure 4 that are labeled "Respond A." Consideration of symmetry indicates that the volume within the upper region is equal to that within the lower region so that only one of these regions need be considered. Thus, the false alarm rate for the matching-tosample task when $\beta \geq 1$ is given by the double integral

$$
F=\int_{-\infty}^{\infty} \int_{X_{2\left(\beta, X_{1}\right)}}^{\infty} f_{-d, 0}\left(X_{1}, X_{2}\right)+f_{d^{\prime}, 0}\left(X_{1}, X_{2}\right) d X_{2} d X_{1},
$$

where $f_{\mu_{1}, \mu_{2}}\left(X_{1}, X_{2}\right)$ is the bivariate normal density function with means on the two dimensions, $X_{1}$ and $X_{2}$, of $\mu_{1}$ and $\mu_{2} . X_{2}\left(\beta, X_{1}\right)$ is the likelihood-ratio equation expressed in terms of $X_{2}$ as a function of $\beta$ and $X_{1}$. This function can be expressed most simply in the form 


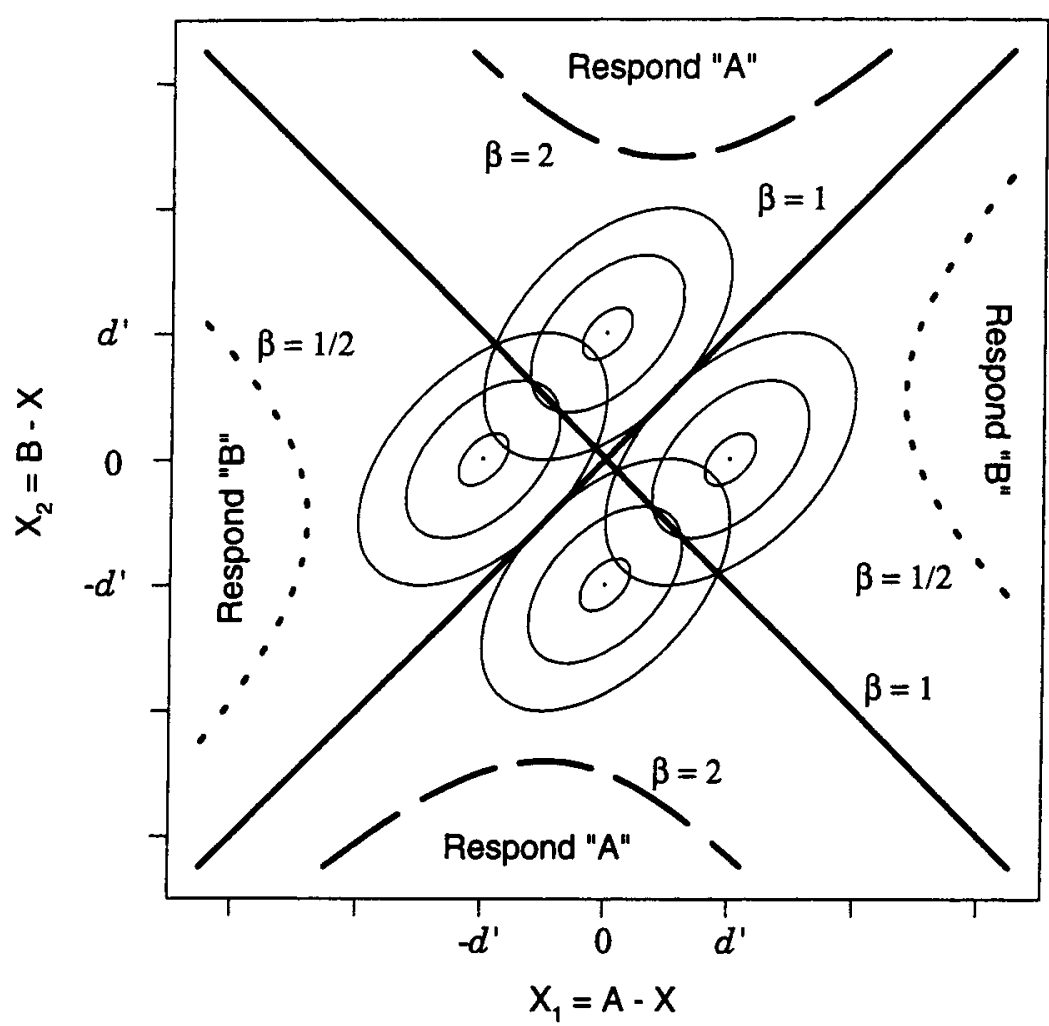

Figure 4. The decision space for the matching-to-sample task for three different decision criteria: $\beta$ equal to $1 / 2,1$, and 2 . If the sensory evidence falls beyond (vertically) the criterial boundaries for $\beta=1$ or 2 , or between the boundaries for $\beta=1 / 2$, the observer responds " $A$ "; otherwise the observer responds " $B$."

$$
\begin{aligned}
\beta\left(X_{1}, X_{2}, d^{\prime}\right) & =\frac{f_{0, d^{\prime}}+f_{0,-d^{\prime}}}{f_{-d^{\prime}, 0}+f_{d^{\prime}, 0}} \\
& =\frac{e^{\frac{1}{3} d^{\prime}\left(X_{1}-X_{2}\right)}\left(e^{\frac{4}{3} d^{\prime} X_{2}}+e^{\frac{2}{3} d^{\prime} X_{1}}\right)}{e^{\frac{4}{3} d^{\prime} X_{1}}+e^{\frac{2}{3} d^{\prime} X_{2}}} .
\end{aligned}
$$

A hit can similarly be defined as the occurrence of Response $\mathrm{A}$ when the correct response was in fact $\mathrm{A}$. Therefore the hit rate is equal to the proportion of the volume under the two distributions with centroids at $X_{1}=0$ that falls within the regions in Figure 4 that are labeled "Respond A." Thus, again considering symmetry, the hit rate for the matching-to-sample task when $\beta \geq 1$ is given by the double integral

$$
H=\int_{-\infty}^{\infty} \int_{X_{2}\left(\beta, X_{1}\right)}^{\infty} f_{0, d^{\prime}}\left(X_{1}, X_{2}\right)+f_{0,-d^{\prime}}\left(X_{1}, X_{2}\right) d X_{2} d X_{1}
$$

When $\beta<1$, the integration in Equations 11 and 13 must be done in a different order because, as can be seen in Figure 4 for the case of $\beta=1 / 2$, the distance between the two criterial boundaries cannot be represented in terms of $X_{2}$. The correct equations are obtained by substituting $X_{1}\left(\beta, X_{2}\right)$ for $X_{2}\left(\beta, X_{1}\right)$ and integrating over $X_{1}$ followed by $X_{2}$ (i.e., exchanging $\mathrm{d} X_{2}$ and $\mathrm{d} X_{1}$ in Equations 11 and 13). The result is the complement of the false alarm and hit rates for $\beta<1$ because it is the volume between the criterial boundaries that is of interest for this case.

Given this information, $\operatorname{VAR}\left(\hat{d}^{\prime}\right)$ and $\operatorname{MSD}\left(\hat{d}^{\prime}\right)$ can be calculated exactly by using the same method described in the section on the same-different task - that is, by calculating the desired statistics from the probability of occurrence of each possible value of $\hat{d}^{\prime}$ that can be sampled for a specified $d^{\prime}, \beta$ and $N$.

\section{Comparing Model Efficiency}

The value of VAR $\left(\hat{d}^{\prime}\right)$ obtained for different tasks, using the same number of trials for each task, gives an indication of the efficiency of each task. However, as has been demonstrated, $\operatorname{VAR}\left(\hat{d}^{\prime}\right)$ does not take into consideration the bias introduced into the estimation process by corrections for extreme proportions. Also, $\operatorname{VAR}\left(\hat{d}^{\prime}\right)$ can be shown to increase as a function of $N$. This implies that conducting more trials actually increases the uncertainty in the estimated parameter. Therefore, $\operatorname{MSD}\left(\hat{d}^{\prime}\right)$ may be a more prudent statistic to consider when choosing a task to employ in a specific context. Figures 5-9 illustrate VAR $\left(\hat{d}^{\prime}\right)$ (thin lines) and $\operatorname{MSD}\left(\hat{d}^{\prime}\right)$ (thick lines) for the four tasks that have been discussed, each at five different levels of sensitivity: $d^{\prime}=0.5,1.0,2.0,3.0$, and 4.0. Each curve is based 


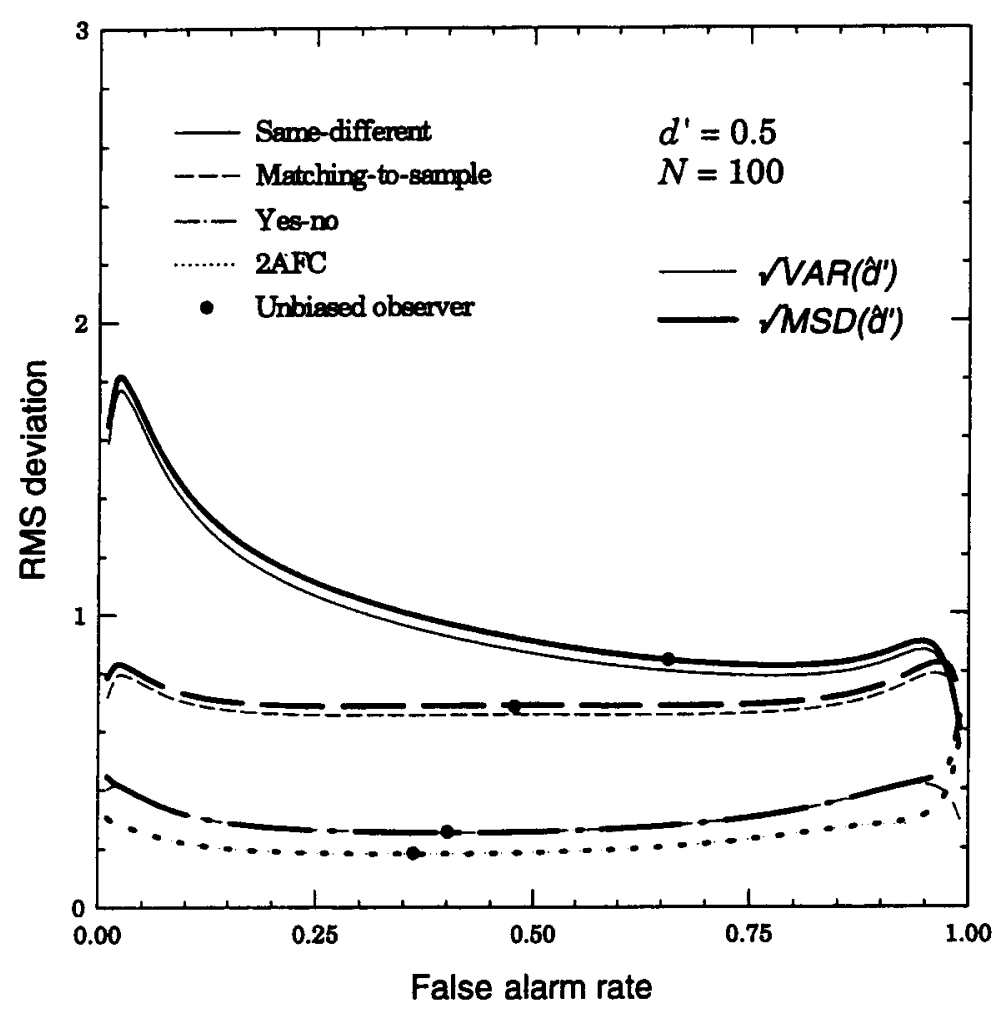

\begin{abstract}
Figure 5. VAR $\left(\hat{d}^{\prime}\right)$ (thin lines) and $\operatorname{MSD}\left(\hat{d}^{\prime}\right)$ (thick lines) as a function of the criterion specified in terms of the population parameter, $F$. All values are based on the log-linear correction, $N=100$, and $d^{\prime}=0.5$. Solid points have been placed at the criterion adopted by an unbiased observer.
\end{abstract}

on $N=100$ trials. The lower on the graph that a point on a $\operatorname{VAR}\left(\hat{d}^{\prime}\right)$ curve lies, the more efficient is that task under the conditions of bias and discriminability specified by that point; and the lower a point lies on an $\operatorname{MSD}\left(\hat{d}^{\prime}\right)$ curve, the more efficient and/or less biased is that task under the conditions specified by that point. The solid points on the curves represent the criterion, in terms of the false alarm rate, that would be adopted by an unbiased observer on that task (i.e., $\beta=1$ ). Moving from left to right within each figure, the points correspond to the following tasks: $2 \mathrm{AFC}$, yes-no, matching-to-sample, and same-different.

When $d^{\prime}=0.5$ (Figure 5), the yes-no and 2AFC tasks yield values of $\operatorname{VAR}\left(\hat{d}^{\prime}\right)$ and $\operatorname{MSD}\left(\hat{d}^{\prime}\right)$ that are lower than those obtained for the other two tasks. Also, $\operatorname{VAR}\left(\hat{d}^{\prime}\right) \simeq$ $\operatorname{MSD}\left(\hat{d}^{\prime}\right)$ for the yes-no and 2AFC tasks over almost the entire domain of possible response biases $\left(\operatorname{MSD}\left(\hat{d}^{\prime}\right) \leq 1.01\right.$ $\times \operatorname{VAR}\left(\hat{d}^{\prime}\right)$ when $.03<F<.89$ for the 2 AFC task, and when $.03<F<.92$ for the yes-no task). This suggests that $\mathrm{E}\left(\hat{d}^{\prime}\right)$ converges to $d^{\prime}$ more rapidly as $N$ increases for these two tasks at low levels of discriminability. These findings suggest that the sensitivity of an observer to barely discriminable events could best be determined using either of these two tasks. If the number of stimulus presentations must be kept to a minimum, then the yes-no task would be the best choice. This is particularly true if several estimates of $d^{\prime}$ obtained using the yes-no task are to be compared. However, caution must be taken when comparing estimates of $d^{\prime}$ obtained from a yes-no task with those obtained from other tasks because empirical results indicate that estimates of $d^{\prime}$ obtained from the yesno task are often less than those obtained from other tasks (Jesteadt \& Bilger, 1974). In addition, the yes-no task is more prone to biased responding than is the $2 \mathrm{AFC}$ task (Macmillan \& Creelman, 1991, p. 124). If at all possible, the 2AFC task may be a safer choice.

When $d^{\prime}=1.0$ (Figure 6), the shapes of the curves obtained for each task are similar to those obtained for the case of $d^{\prime}=0.5$. Values of $\operatorname{MSD}\left(\hat{d}^{\prime}\right)$ for the $2 \mathrm{AFC}$ and yes-no task are marginally larger when $d^{\prime}=1.0$, whereas values for the matching-to-sample and the same-different tasks are considerably smaller. This highlights a pattern that can be observed across Figures 5-9. MSD $\left(\hat{d}^{\prime}\right)$ increases systematically as a function of $d^{\prime}$ for the yes-no and 2AFC tasks, whereas for the other two tasks, this statistic appears to first decrease and later increase as $d^{\prime}$ increases. This pattern is clarified in Figure 10, which illustrates $\operatorname{MSD}\left(\hat{d}^{\prime}\right)$ (thick lines) and $\operatorname{VAR}\left(\hat{d}^{\prime}\right)$ (thin lines) as a function of $d^{\prime}$ for an unbiased observer in each of the four tasks. Figure 10 reveals an additional feature not apparent in Figures 5-9: There is an initial increase in $\operatorname{MSD}\left(\hat{d}^{\prime}\right)$ at very low levels of discriminability for the same-different and matchingto-sample tasks. 


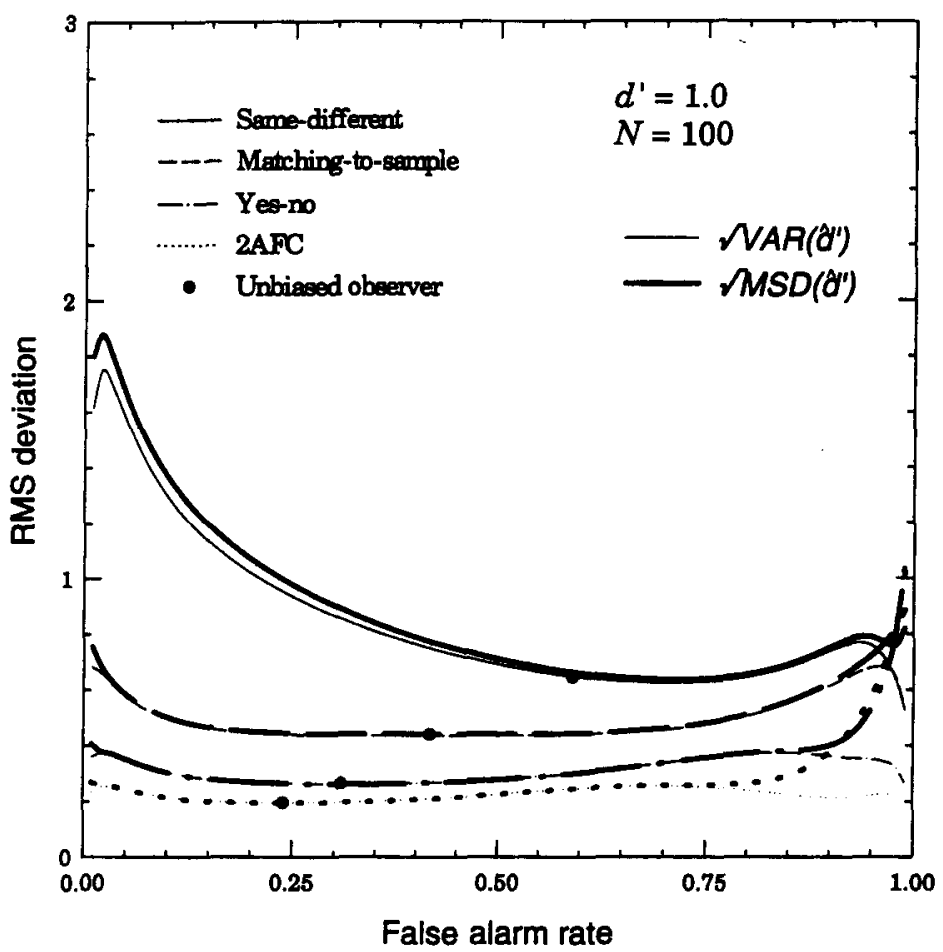

Figure 6. VAR( $\left(\hat{d}^{\prime}\right)$ (thin lines) and $\operatorname{MSD}\left(\hat{d}^{\prime}\right)$ (thick lines) as a function of the criterion specified in terms of the population parameter, $F$. All values are based on the log-linear correction, $N=100$, and $d^{\prime}=1.0$. Solid points have been placed at the criterion adopted by an unbiased observer (i.e., $\beta=1$ ).

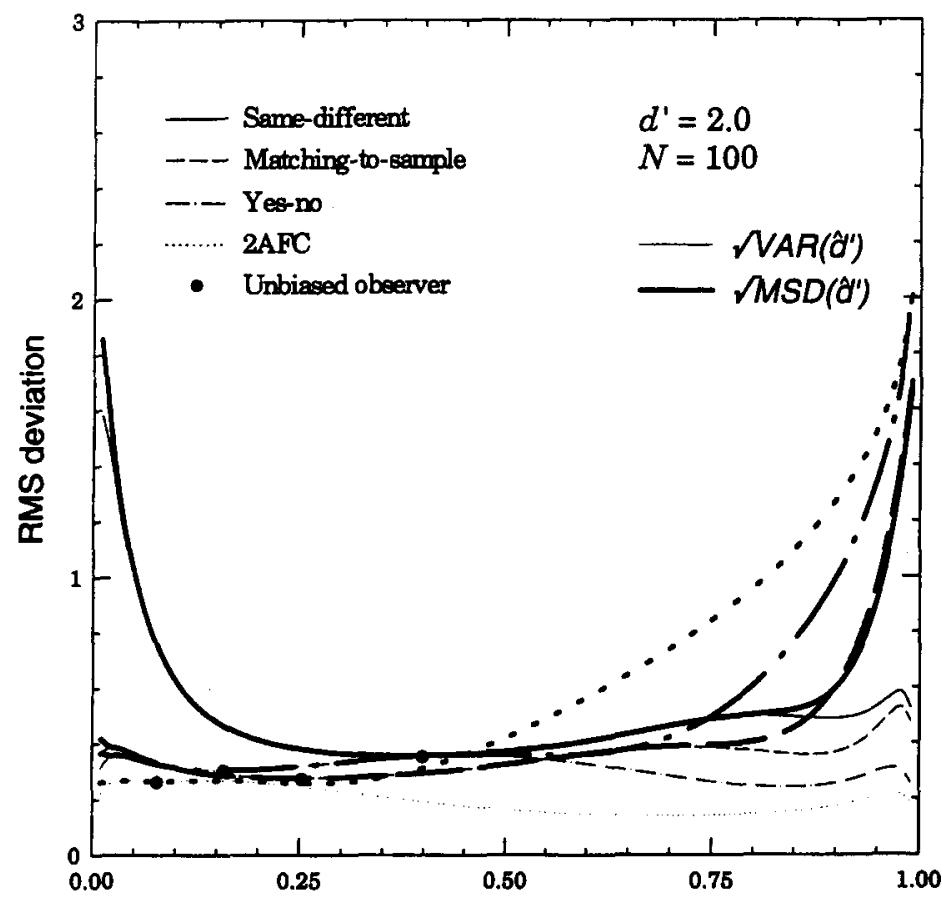

False alarm rate

Figure 7. VAR $\left(\hat{d}^{\prime}\right)$ (thin lines) and $\operatorname{MSD}\left(\hat{d}^{\prime}\right)$ (thick lines) as a function of the criterion specified in terms of the population parameter, $F$. All values are based on the $\log$-linear correction, $N=100$, and $d^{\prime}=\mathbf{2 . 0}$. Solid points have been placed at the criterion adopted by an unbiased observer (i.e., $\beta=1$ ). 


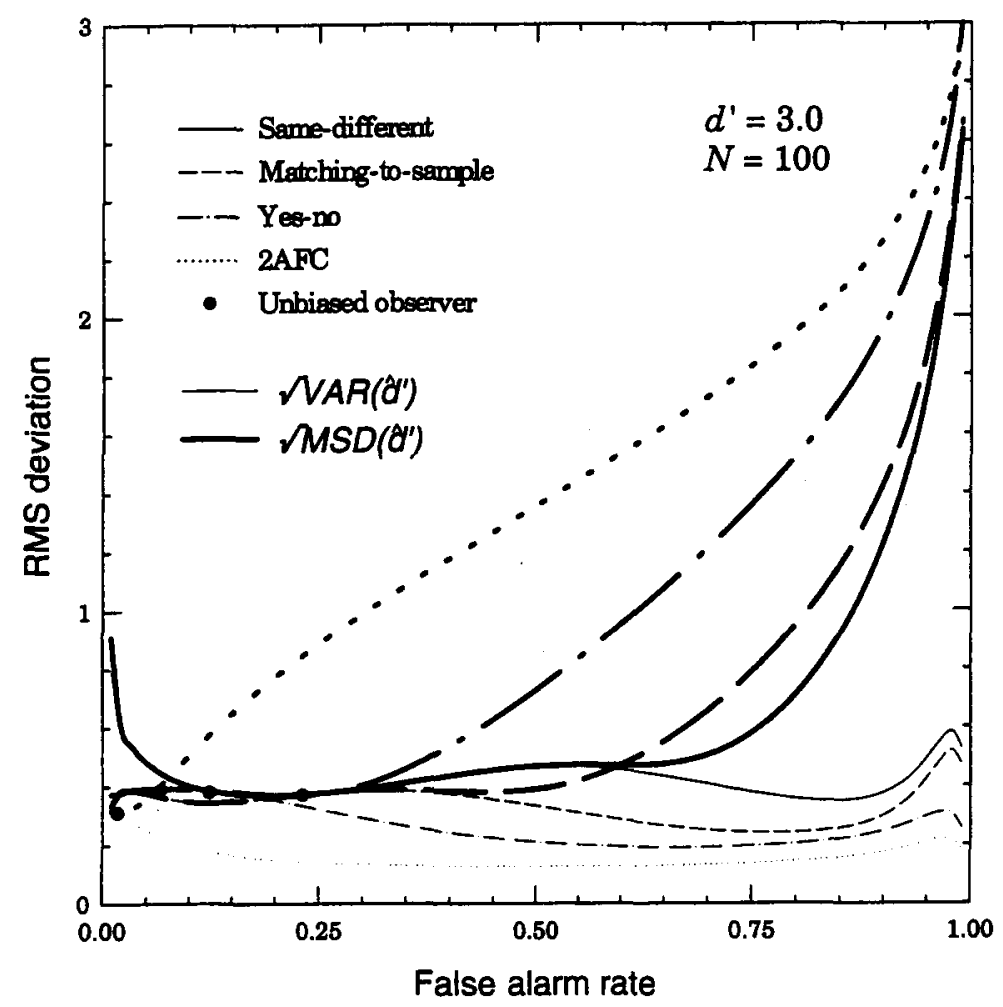

Figure 8. VAR $\left(\hat{d}^{\prime}\right)$ (thin lines) and $\operatorname{MSD}\left(\hat{d}^{\prime}\right)$ (thick lines) as a function of the criterion specified in terms of the population parameter, $F$. All values are based on the log-linear correction, $N=100$, and $d^{\prime}=3.0$. Solid points have been placed at the criterion adopted by an unbiased observer (i.e., $\beta=1$ ).

When $d^{\prime}=2$ (Figure 7), the 2AFC task produces marginally lower values of $\operatorname{MSD}\left(\hat{d}^{\prime}\right)$ than does the matchingto-sample task when $F<.37$ (the median difference is $-6.6 \%$ ). However, the matching-to-sample tasks yields lower values of $\operatorname{MSD}\left(\hat{d}^{\prime}\right)$ than does the $2 \mathrm{AFC}$ task for more extreme criteria. The same-different task gives relatively large values of $\operatorname{MSD}\left(\hat{d}^{\prime}\right)$ for extreme response biases in either direction.

When $d^{\prime}=3$ (Figure 8), the matching-to-sample task yields values of $\operatorname{MSD}\left(\hat{d}^{\prime}\right)$ that are less than those obtained for the other three tasks over the entire criterion domain, except for two cases: (1) The 2AFC task provides slightly lower values of $\operatorname{MSD}\left(\hat{d}^{\prime}\right)$ when the criterion is at a false alarm rate less than .06. (2) The same-different task yields lower values of $\operatorname{MSD}\left(\hat{d}^{\prime}\right)$ for extreme criteria $(F>.59)$. Any fervor for the use of the matching-to-sample task must be tempered with the realization that, for the same number of trials, $50 \%$ more stimulus presentations are required than for the $2 \mathrm{AFC}$ task. Even taking this into account, the matchingto-sample task may be an advantageous choice when $d^{\prime} \simeq$ 3.0.

When $d^{\prime}=4.0$ (Figure 9), the yes-no and 2AFC tasks result in values of $\operatorname{MSD}\left(\hat{d}^{\prime}\right)$ that increase rapidly as the observer becomes more biased. Yet, the yes-no task has the lowest values of $\operatorname{MSD}\left(\hat{d}^{\prime}\right)$ over a small range of criteria $(.02 \leq F \leq .07$; the median difference is $-5.0 \%)$. The matching-to-sample and same-different tasks yield smaller values of $\operatorname{MSD}\left(\hat{d}^{\prime}\right)$ over all other observer biases. The same-different task has the lowest values of $\operatorname{MSD}\left(\hat{d}^{\prime}\right)$ when the criterion is set to a false alarm rate greater than .25 . As the yes-no task provides but a small window of optimality in terms of criterial range, the selection of either of these other two tasks is preferable to either $2 \mathrm{AFC}$ or yes-no.

As illustrated in Figures 1 and 2, for the yes-no task $\operatorname{VAR}\left(\hat{d}^{\prime}\right) \propto 1 / N$ only when $\operatorname{VAR}\left(\hat{d}^{\prime}\right) \simeq \operatorname{MSD}\left(\hat{d}^{\prime}\right)$ - that is, when $N$ is sufficiently large that minimal bias is introduced by the correction rule employed. This indicates that $\operatorname{VAR}\left(\hat{d}^{\prime}\right)$ and $\operatorname{MSD}\left(\hat{d}^{\prime}\right)$ cannot be reliably calculated from the values indicated in Figures $5-9$ by simply multiplying the obtained value by a constant that is proportional to the $N$ of interest. There is insufficient space here to produce multiple figures; however, the relative efficiencies of these tasks when the observer is unbiased (i.e., $\beta=1$ ) are given in Table 1. The values in the table are the relative efficiencies, in terms of the ratio of $\operatorname{VAR}\left(\hat{d}^{\prime}\right)$, of the yes-no task relative to the remaining three tasks [ratios are also specified for values of $\left.\operatorname{MSD}\left(\hat{d}^{\prime}\right)\right]$. A value greater than one indicates an efficiency greater than that of the yes-no task. To obtain the relative efficiency of any two tasks in the table, divide the respective values in the table. Thus, the relative efficiency of the 2 AFC task with respect to the same- 


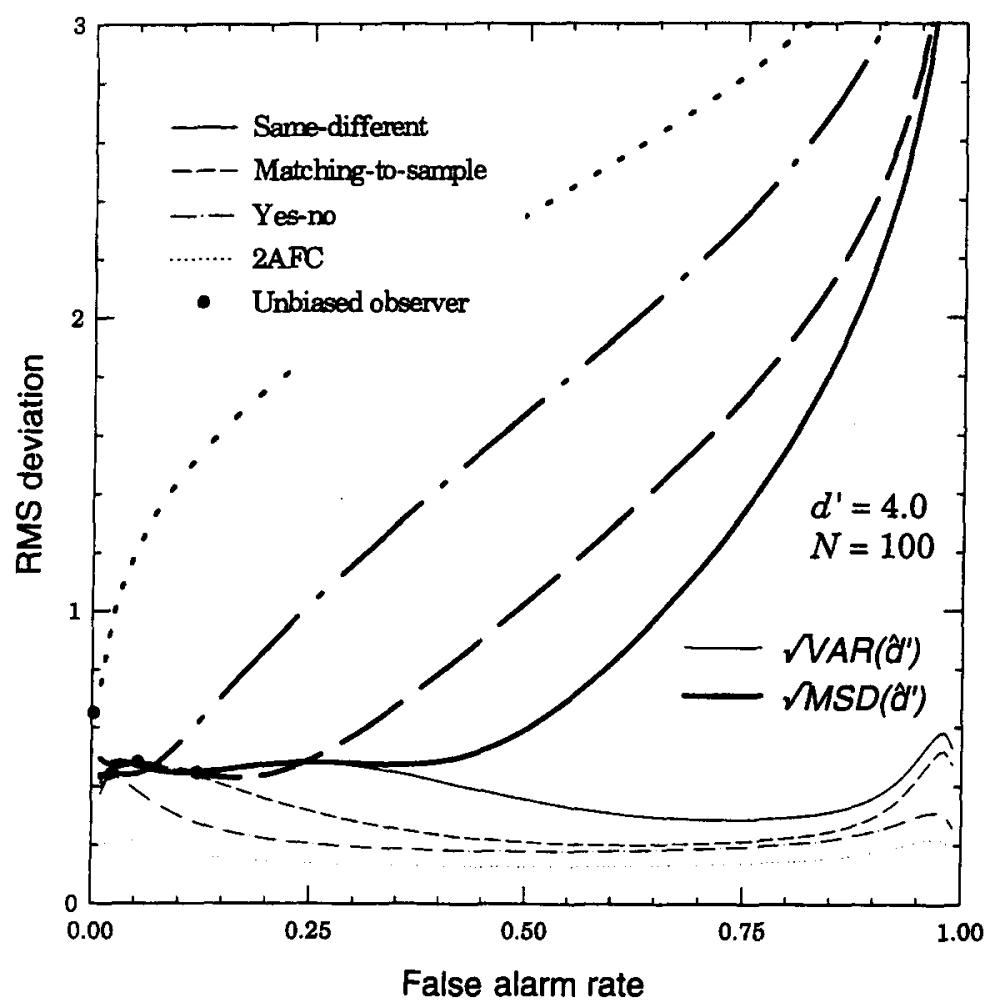

Figure 9. $\operatorname{VAR}\left(\hat{d}^{\prime}\right)$ (thin lines) and $\operatorname{MSD}\left(\hat{d}^{\prime}\right)$ (thick lines) as a function of the criterion specified in terms of the population parameter, $F$. All values are based on the log-linear correction, $N=100$, and $d^{\prime}=4.0$. Solid points have been placed at the criterion adopted by an unbiased observer (i.e., $\beta=1$ ).

different task [in terms of $\operatorname{VAR}\left(d^{\prime}\right)$ ] when $d^{\prime}=2$ and $N=$ 100 is $1.32 \div 0.73=1.81$; that is, the $2 \mathrm{AFC}$ task is about 1.8 times more efficient than the same-different task under these circumstances.

A cautionary note is required here. The values in Table 1 are correct for unbiased observers. However, Figures 5-9 indicate that $\operatorname{MSD}\left(d^{\prime}\right)$ can increase very rapidly as the observer becomes more biased. This is particularly notable for higher levels of discriminability. For example, when $d^{\prime}=$ 3 and $N=100$ (see Figure 8 ), $\operatorname{MSD}\left(\hat{d}^{\prime}\right)$ is larger for the $2 \mathrm{AFC}$ task than for any of the other tasks when the criterion is set above $F=.08$. However, Table 1 indicates (and

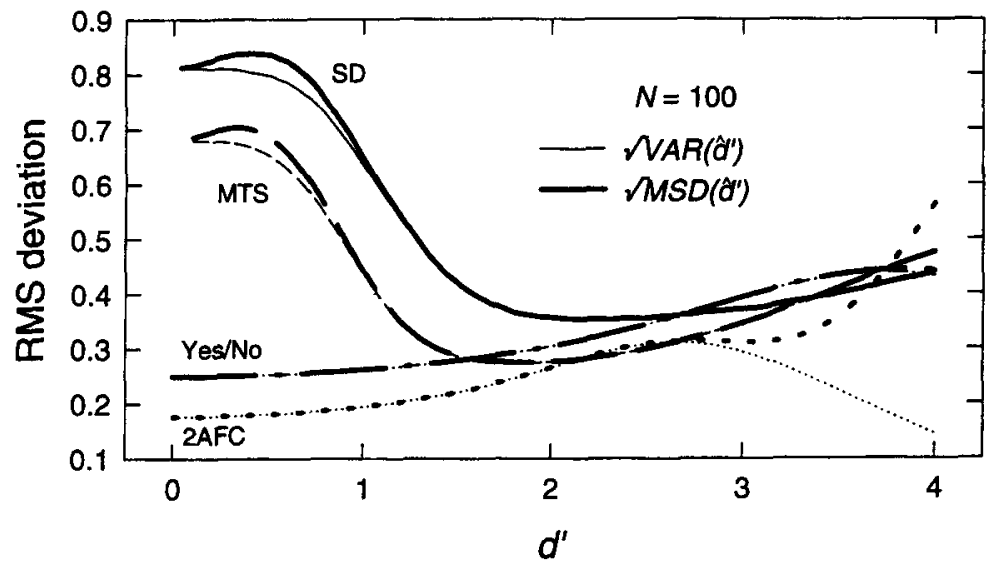

Figure 10. $\operatorname{VAR}\left(\hat{d}^{\prime}\right)$ (thin lines) and $\operatorname{MSD}\left(\hat{d}^{\prime}\right)$ (thick lines) as a function of $d^{\prime}$ for each of the four tasks considered. The observers are assumed to be unbiased. Notice how $\operatorname{VAR}\left(\hat{d}^{\prime}\right)$ initially decreases at low levels of discriminability for the samedifferent and matching-to-sample tasks. This is not due to ceiling effects. 
Table 1

Relative Efficiencies [in Terms of $\operatorname{VAR}\left(\hat{d}^{\prime}\right)$ and $\left.\operatorname{MSD}\left(\hat{d}^{\prime}\right)\right]$ for Three Tasks Specified With Respect to the Yes-No Task

\begin{tabular}{|c|c|c|c|c|c|c|c|c|c|}
\hline \multirow[b]{2}{*}{$d^{\prime}$} & \multirow[b]{2}{*}{ Task } & \multicolumn{2}{|c|}{$N=10$} & \multicolumn{2}{|c|}{$N=30$} & \multicolumn{2}{|c|}{$N=100$} & \multicolumn{2}{|c|}{$N=300$} \\
\hline & & $\operatorname{VAR}\left(\hat{d}^{\prime}\right)$ & $\operatorname{MSD}\left(\hat{d}^{\prime}\right)$ & $\operatorname{VAR}\left(\hat{d}^{\prime}\right)$ & $\operatorname{MSD}\left(\hat{d}^{\prime}\right)$ & $\operatorname{VAR}\left(\hat{d}^{\prime}\right)$ & $\overline{\operatorname{MSD}\left(\hat{d}^{\prime}\right)}$ & $\operatorname{VAR}\left(\hat{d}^{\prime}\right)$ & $\operatorname{MSD}\left(\hat{d}^{\prime}\right)$ \\
\hline \multirow[t]{3}{*}{0.5} & $2 \mathrm{AFC}$ & 2.01 & 2.01 & 1.95 & 1.95 & 1.95 & 1.95 & 1.96 & 1.96 \\
\hline & SD & 0.25 & 0.23 & 0.16 & 0.15 & 0.10 & 0.09 & 0.06 & 0.06 \\
\hline & MTS & 0.37 & 0.34 & 0.24 & 0.22 & 0.15 & 0.14 & 0.39 & 0.39 \\
\hline \multirow[t]{3}{*}{1.0} & $2 \mathrm{AFC}$ & 2.10 & 2.08 & 1.81 & 1.81 & 1.82 & 1.82 & 1.82 & 1.82 \\
\hline & SD & 0.25 & 0.23 & 0.20 & 0.18 & 0.16 & 0.16 & 0.21 & 0.21 \\
\hline & MTS & 0.39 & 0.36 & 0.34 & 0.32 & 0.36 & 0.35 & 0.56 & 0.55 \\
\hline \multirow[t]{3}{*}{2.0} & $2 \mathrm{AFC}$ & 3.08 & 2.08 & 1.96 & 1.90 & 1.32 & 1.31 & 1.34 & 1.34 \\
\hline & SD & 0.27 & 0.28 & 0.54 & 0.54 & 0.73 & 0.73 & 0.75 & 0.75 \\
\hline & MTS & 0.56 & 0.57 & 1.05 & 1.05 & 1.21 & 1.21 & 1.22 & 1.22 \\
\hline \multirow[t]{3}{*}{3.0} & $2 \mathrm{AFC}$ & 6.62 & 0.74 & 4.57 & 1.40 & 1.79 & 1.60 & 0.81 & 0.80 \\
\hline & SD & 0.26 & 0.46 & 0.61 & 0.65 & 1.11 & 1.11 & 1.11 & 1.11 \\
\hline & MTS & 0.59 & 0.85 & 0.83 & 0.87 & 1.29 & 1.29 & 1.32 & 1.32 \\
\hline \multirow[t]{3}{*}{4.0} & $2 \mathrm{AFC}$ & 18.18 & 0.51 & 15.29 & 0.36 & 8.91 & 0.62 & 2.75 & 1.13 \\
\hline & SD & 0.16 & 1.15 & 0.30 & 0.66 & 0.94 & 0.98 & 1.67 & 1.67 \\
\hline & MTS & 0.43 & 1.01 & 0.48 & 0.88 & 0.83 & 0.86 & 1.38 & 1.38 \\
\hline
\end{tabular}

Note-Values reflect the performance of an unbiased observer in each task. Values greater than unity indicate efficiency greater than that of the yes-no task at the same level of discriminability. 2AFC, twoalternative forced-choice; SD, same-different; MTS, matching-to-sample.

Figure 8 shows) that, for an unbiased observer, $\operatorname{MSD}\left(\hat{d}^{\prime}\right)$ is the lowest for the 2AFC task.

\section{Summary}

When deciding which psychophysical task to employ in an empirical study, the efficiency of available tasks, specified in a manner that incorporates estimator bias and under the conditions likely to be encountered during the study, should be taken into consideration. For barely discriminable events ( $d^{\prime}$ less than about one half), the yes-no and 2AFC tasks are the best choice. For highly discriminable events ( $d^{\prime}$ greater than about three), same-different and matching-to-sample tasks may be advantageous.

It is also important to encourage unbiased responding from the subject whenever possible. This is because values of $\operatorname{MSD}\left(\hat{d}^{\prime}\right)$ for all tasks considered in this paper were near their lowest in the vicinity of the criterion that would be adopted by an unbiased observer. ${ }^{4}$

If an investigator selects a task that has a low likelihood of extreme probabilities when employed in the experimental context, then it is likely that the task is fairly efficient for use in that context. ${ }^{5}$ However, it is apparent that a reduction in the occurrence of extreme proportions is in itself not a guarantee of high efficiency. This was clearly evident from the low efficiency of the matching-to-sample and same-different tasks at low values of $d^{\prime}$ when the observer was unbiased.

A general rule of thumb is to choose the task with the lowest value of $\operatorname{MSD}\left(\hat{d}^{\prime}\right)$ for the level of discriminability to be investigated and to encourage unbiased responding. These findings indicate that each of the tasks investigated in this study has value in specific circumstances and, hence, any temptation to settle for a favorite task should be avoided.

\section{REFERENCES}

BERLINER, J. E., \& DURLACH, N. I. (1973). Intensity perception: IV. Resolution in roving-level discrimination. Journal of the Acoustical Society of America, 53, 1270-1287.

Goodman, L. A. (1970). The multivariate analysis of qualitative data: Interactions among multiple classifications. Journal of the American Statistical Association, 65, 226-256.

GREEN, D. M., \& SwETS, J. A. (1966). Signal detection theory and psychophysics. New York: Wiley.

HaUTUS, M. J. (1995). Corrections for extreme proportions and their biasing effects on estimated values of $d^{\prime}$. Behavior Research Methods, Instruments, \& Computers, 27, 47-51.

JESTEADT, W., \& BILGER, R. C. (1974). Intensity and frequency discrimination in one- and two-interval paradigms. Journal of the Acoustical Society of America, 55, 1266-1276.

Kaplan, H. L., Macmillan, N. A., \& Creelman, C. D. (1978). Tables of $d^{\prime}$ for variable-standard discrimination paradigms. Behavior Research Methods \& Instrumentation, 10, 796-813.

Macmillan, N. A., \& Creelman, C. D. (1991). Detection theory: A user's guide. New York: Cambridge University Press.

Macmillan, N. A., \& Kaplan, H. L. (1985). Detection theory analysis of group data: Estimating sensitivity from average hit and false alarm rates. Psychological Bulletin, 98, 185-199.

Macmillan, N. A., Kaplan, H. L., \& Creelman, C. D. (1977). The psychophysics of categorical perception. Psychological Review, 84, 452-471.

MilleR, J. (1996). The sampling distribution of $d^{\prime}$. Perception \& Psychophysics, 58, 65-72.

NOREEN, D. L. (1981). Optimal decision rules for some common psychophysical paradigms. In S. Grossberg (Ed.), Mathematical psychology and psychophysiology: Seminars in applied mathematics (Vol. 13, pp. 237-280). Providence, RI: American Mathematical Society.

Pierce, J. R., \& Gilbert, E. N. (1958). On AX and ABX limens. Journal of the Acoustical Society of America, 30, 593-595.

SNODGRASs, J. G., \& CoRwIN, J. (1988). Pragmatics of measuring recognition memory: Applications to dementia and amnesia. Journal of Experimental Psychology: General, 117, 34-50.

SorkIN, R. D. (1962). Extension of the theory of signal detectability to matching procedures in psychoacoustics. Journal of the Acoustical Society of America, 34, 1745-1751. 


\section{NOTES}

1. The biases obtained using Equation 3 agree closely with those reported by Hautus (1995) that were obtained with Monte Carlo methods.

2. This strategy can be contrasted with the optimal or likelihood-ratio decision strategy (Noreen, 1981).

3. An equation in which the variable of interest cannot be written as an explicit function of the remaining variables; that is, the variable of interest cannot be isolated on one side of the equality sign.

4. For the yes-no task, the point on the ROC obtained by an unbiased observer (i.e., $\beta=1$ ) falls on the negative diagonal, which has the lowest likelihood of extreme proportions. For other tasks, such as the same-different task, an unbiased observer will produce a point that is not on the negative diagonal. Thus, for such other tasks, the least estimator bias will be obtained for an observer exhibiting some response bias.

5. As indicated earlier, this is the case when $\operatorname{VAR}\left(\hat{d}^{\prime}\right) \simeq \operatorname{MSD}\left(\hat{d}^{\prime}\right)$.

\section{APPENDIX}

If the $1 /(2 N)$ correction is applied to the binomially distributed sample proportions, then the expected value of $z(\hat{p})$ is given by

$$
\begin{aligned}
\mathrm{E}(z(\hat{p}))= & \sum_{x=1}^{N_{p}-1}\left[z\left(\frac{x}{N_{p}}\right) b\left(x, N_{p}, p\right)\right] \\
& +z\left(1-\frac{1}{2 N_{p}}\right) p^{N_{p}}+z\left(\frac{1}{2 N_{p}}\right)(1-p)^{N_{p}}
\end{aligned}
$$

It therefore follows that

$$
\begin{aligned}
\mathrm{E}\left(\hat{d}^{\prime}\right)= & \mathrm{E}(z(\hat{H}))-\mathrm{E}(z(\hat{F})) \\
= & \sum_{x=1}^{N_{s}-1}\left[z\left(\frac{x}{N_{s}}\right) b\left(x, N_{s}, H\right)\right] \\
& +z\left(1-\frac{1}{2 N_{s}}\right) H^{N_{s}}+z\left(\frac{1}{2 N_{s}}\right)(1-H)^{N_{s}}
\end{aligned}
$$

$$
\begin{aligned}
& -\left[\sum_{x=1}^{N_{n}-1}\left[z\left(\frac{x}{N_{n}}\right) b\left(x, N_{n}, F\right)\right]\right. \\
& \left.\quad+z\left(1-\frac{1}{2 N_{n}}\right) F^{N_{n}}+z\left(\frac{1}{2 N_{n}}\right)(1-F)^{N_{n}}\right],
\end{aligned}
$$

and hence the exact variance of $\hat{d}^{\prime}$ given the application of the $1 /(2 \mathrm{~N})$ correction is

$$
\begin{aligned}
\operatorname{VAR}\left(\hat{d}^{\prime}\right)= & \sum_{x=1}^{N_{s}-1}\left[\left[z\left(\frac{x}{N_{s}}\right)-\mathrm{E}(z(\hat{H}))\right]^{2} b\left(x, N_{s}, H\right)\right] \\
& +\left[z\left(\frac{1}{2 N_{s}}\right)-\mathrm{E}(z(\hat{H}))\right]^{2}(1-H)^{N_{s}} \\
& +\left[z\left(1-\frac{1}{2 N_{s}}\right)-\mathrm{E}(z(\hat{H}))\right]^{2} H^{N_{s}} \\
& +\sum_{x=1}^{N_{n}-1}\left[\left[z\left(\frac{x}{N_{n}}\right)-\mathrm{E}(z(\hat{F}))\right]^{2} b\left(x, N_{n}, F\right)\right] \\
& +\left[z\left(\frac{1}{2 N_{n}}\right)-\mathrm{E}(z(\hat{F}))\right]^{2}(1-F)^{N_{n}} \\
& +\left[z\left(1-\frac{1}{2 N_{n}}\right)-\mathrm{E}(z(\hat{F}))\right]^{2} F^{N_{n}}
\end{aligned}
$$

$\operatorname{MSD}\left(\hat{d}^{\prime}\right)$ is obtained from Equation $\mathrm{A} 3$ by substituting $z(H)$ for $\mathrm{E}(z(\hat{H}))$ and $z(F)$ for $\mathrm{E}(z(\hat{F}))$. This is analogous to the steps taken to obtain Equation 6 for the log-linear correction.

(Manuscript received October 10, 1994; revision accepted for publication May 12, 1997.) 